\title{
68. A YOUNG ASSOCIATION IN THE LARGE MAGELLANIC CLOUD
}

\author{
B. J. BoK \\ Mount Stromlo Observatory
}

During the past three years, Priscilla F. Bok, Jane Basinski, and I have concentrated in our work on the Large Magellanic Cloud upon the study of young associations. One colour-magnitude array, that of NGC 1955-68-74, has been published (Bok, Bok, and Basinski 1962), and work on three additional groupings, NGC 1929-34-35-36-37, NGC 1770, and NGC 1814-16-20 is well under way. We shall report here on NGC 1929-37, which is often referred to as "Shapley's Constellation I'. Its position is $\alpha=5^{\mathrm{h}} 22^{\mathrm{m}} .0 ; \delta=-68^{\circ} 00^{\prime}(1960)$.

NGC 1929-37 is one of the more conspicuous, isolated associations (Plate 1). It is more brilliant, and somewhat larger, than average and hence it is an ideal object for detailed study. While the nebulosity is strong in the ultraviolet and in the light of the nebular lines, its effects can be eliminated almost entirely by a suitable choice of photographic emulsions and filters. The integrated properties of the association are (Bok and Bok 1962):

$$
V=8 \cdot 46 ; B-V=-0 \cdot 06 ; U-B=-0 \cdot 99 .
$$

Since $A_{V}=0^{\mathrm{m}} \cdot 7$, we have

$$
(B-V)_{0}=-0 \cdot 29 ;(U-B)_{0}=-1 \cdot 15 .
$$

On the assumption of a distance modulus for the Large Cloud $(m-M)_{0}=19 \cdot 0$ perhaps on the large side-we find the value $M_{0}=-11 \cdot 2$ for the integrated visual absolute magnitude of the object; at the assumed distance, $1^{\prime}=19 \mathrm{pc}$.

\section{The Colour-Magnitude Array}

The colour-magnitude array for Constellation I is shown in Figures $1(a)$ and $1(b)$. Figure 1 $(a)$ shows the array for the central area under investigation (43 square minutes of arc), Figure $1(b)$ that for the outer area of 173 square minutes of arc. One should bear in mind that all values of $B-V$ plotted in Figures $1(a)$ and $1(b)$ should be corrected for interstellar reddening by -0.23 on the average; individual corrections of this amount are not justified, since these may show considerable scatter with respect to the mean. The obscuring matter is obviously associated with NGC 1929-37.

Figure 1 $(a)$ shows clearly the apparent absence of red supergiant stars belonging to Constellation I. There are two bright red stars close to the association, HD 35665 and HDE 269448. Their Henry Draper spectral types are respectively G5 and K0. The first star has been listed as a foreground star by the Radcliffe observers, Feast, Thackeray, and Wesselink (1960). The radial velocity for HDE 269448 has been measured by H. F. Gollnow, M. Mowat, and the present author, who find it to be equal to $-33 \mathrm{~km} / \mathrm{sec}$. Apparently neither star is a member of the Large Cloud. It is obviously important to check on the Magellanic Cloud membership of the four stars with $+0 \cdot 4<B-V<+1 \cdot 2$, especially on the one star with $V<13 \cdot 0$. 
But, even if one or more of these stars proves to belong to the Large Cloud, they may not be members of the association proper. The available evidence speaks against the presence of any red supergiants in NGC 1929-37, at least for $M<-4 \cdot 0$. The evolutionary significance of this conclusion is considerable and deserves further consideration.

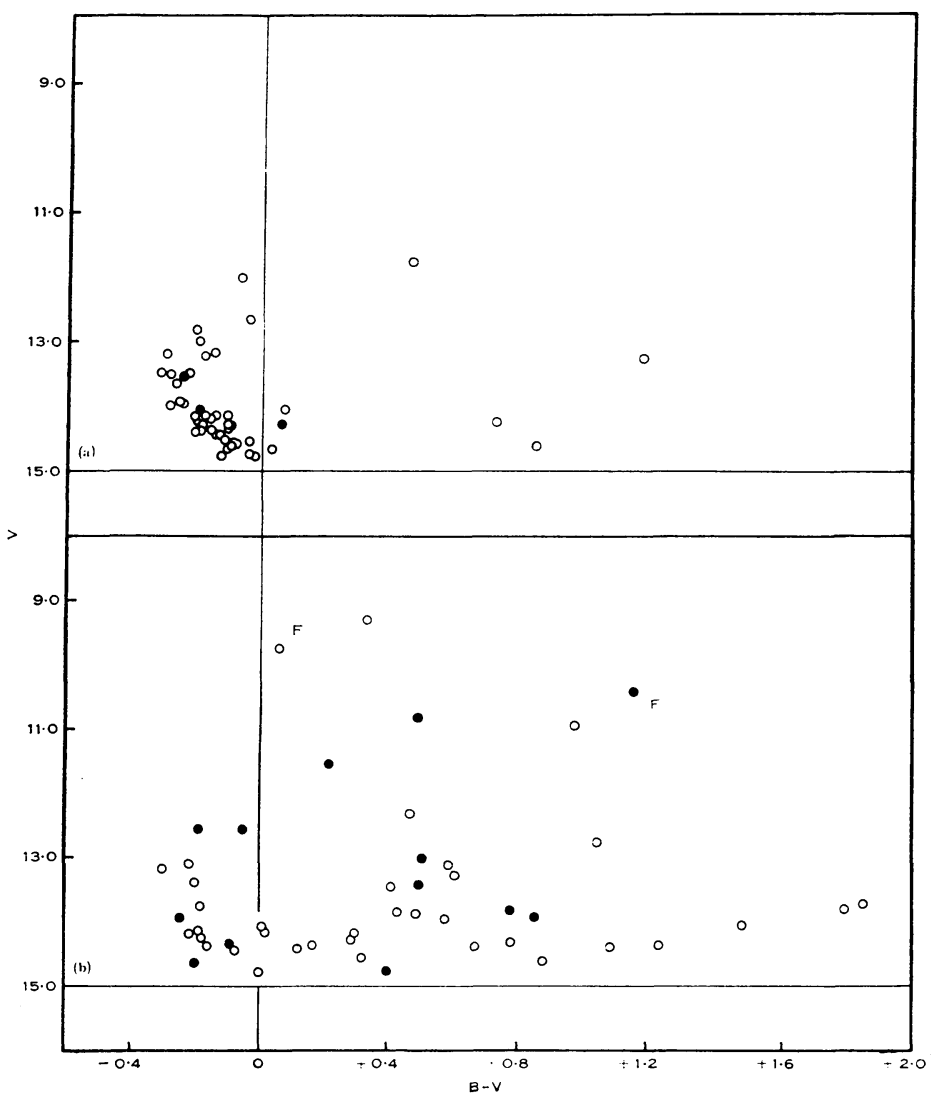

Fig. 1.-Colour-magnitude array for the core of NGC 1929-37 and for a larger area directly surrounding the core. The observed values of $(B-V)$ have not been corrected for the effects of interstellar reddening. (a) Inner region, area c. 43 square minutes; (b) outer region, area c. 173 square minutes.

\section{Total Mass of Stars and Diameter of Association}

In estimating the total mass of the stars belonging to NGC 1929-37, we shall follow the approach of Westerlund (1961) and we shall use the mass-luminosity relation as given by Limber (1960). Star counts have been made by Mrs. Basinski for the field of NGC 1929-37 and for a comparison field. After application of the most likely corrections for field stars, we find a value $M=4000 M_{\odot}$ for the stars with $M \leqslant-4 \cdot 0$ which belong to the association. Westerlund, following Blaauw (1958), has estimated that the above figure represents only about one-sixth of the 
total mass, but there is no direct evidence on this score. Plates (74-inch) of relatively long exposure times do not show any marked crowding of faint stars and off-hand the factor 6 seems to be rather large. The maximum total mass of the stars in NGC 1929-37 equals $2.4 \times 10^{4} M_{\odot}$. All stars that are included in this estimate fall within a distance of $4^{\prime}$ of the centre of the group, to which corresponds a distance of $75 \mathrm{pc}$ at the assumed distance for the Large Cloud of $63,000 \mathrm{pc}$ or $(m-M)_{0}=$ 19.0. The effective diameter to which the mass estimate refers is probably close to $150 \mathrm{pc}$.

The details of the calculations are shown in Table 1.

TABLE 1

MASS ESTIMATE OF STARS IN THREE AREAS

\begin{tabular}{c|c|c|c|c}
\hline$V_{0}$ & $M$ & $\begin{array}{r}\text { Assumed } \\
\text { Mass per Star } \\
\text { (solar masses) }\end{array}$ & $a(m)$ & $\begin{array}{c}a(m) \times \text { Mass } \\
\text { (solar } \\
\text { masses) }\end{array}$ \\
\hline $11 \cdot 0-12 \cdot 0$ & $-7 \cdot 5$ & 200 & 6 & 1200 \\
$12 \cdot 0-13 \cdot 0$ & $-6 \cdot 5$ & 100 & 10 & 1000 \\
$13 \cdot 0-14 \cdot 0$ & $-5 \cdot 5$ & 50 & 25 & 1250 \\
$14 \cdot 0-15 \cdot 0$ & $-4 \cdot 5$ & 20 & 23 & 460 \\
\hline
\end{tabular}

\section{Total Mass of Ionized Hydrogen Estimated from Optical Data}

The following estimates have been prepared by Mr. D. J. Faulkner of Mount Stromlo Observatory:

Doherty, Henize, and Aller (1956) have published tables from which crosssectional profiles of the $\mathrm{H} \alpha$ radiation of the nebula may be constructed (the $\mathrm{H} \alpha$ intensity in units of $\mathrm{erg} / \mathrm{cm}^{2}$.sec.steradian). From these profiles and the angular dimensions of the nebula, it is possible to estimate its total $\mathrm{H}_{\alpha}$ emission in ergs/sec. Assuming the nebula uniform, we can then use its linear dimensions to obtain the $\mathrm{H} \alpha$ emission per unit volume, $E$, which is related to the number density of hydrogen ions by

$$
E=3 \cdot 33 \times 10^{-25} \mathrm{~N}\left(\mathrm{H}^{+}\right)^{2} \mathrm{erg} / \mathrm{sec}^{\mathrm{cm}} \mathrm{cm}^{3}
$$

(for a nebula optically thick in Lyman radiation and of $T_{e}=10,000^{\circ} \mathrm{K}$ ). The derived value of $N\left(\mathrm{H}^{+}\right)$and the dimensions then yield an estimate of the total mass of ionized hydrogen. Note that the above assumption of a uniform nebula means that our mass estimate is an upper limit. The derived value for the total mass of the nebula (ionized hydrogen only) is:

$$
\text { NGC 1929-37 (total) : } 6 \times 10^{4} M_{\odot} \text {. }
$$

Three separate $\mathrm{H} \alpha$ profiles were used to obtain this estimate.

\section{Total Mass of Ionized Hydrogen Estimated from Radio Continuum Data}

Dr. D. S. Mathewson has kindly provided the information that follows from his $1408 \mathrm{Mc} / \mathrm{s}$ observations at Parkes. The association stands out as a marked 
concentration in his contour diagram shown in paper 54 of this volume. He has estimated that the upper limit of the total mass of the ionized hydrogen is:

$$
\text { NGC 1929-37 (total) : } 5 \times 10^{4} M \odot \text {. }
$$

We note that the agreement between the Faulkner (optical) and Mathewson (radio) estimates is an excellent one. The Mathewson estimates refer to an approximate diameter of about $400 \mathrm{pc}$, considerably greater than the principal region of optical $\mathrm{H} \alpha$ emission.

\section{Total Mass of Neutral Hydrogen Estimated from 21-Cm Data}

Mr. R. X. McGee has been good enough to provide the information that follows from his $21-\mathrm{cm}$ observations at Parkes. Rather surprisingly-in view of the abundance of $\mathrm{OB}$ supergiants in the association-the region of NGC 1929-37 stands out as one of the three strongest HI concentrations outside the 30 Doradus complex. It is shown clearly at the centre of the contour diagram shown in paper 62 of the present volume.

The precise estimation of the mass of the HI cloud from McGee's data is made difficult by the fact that the observed concentration stretches southward from NGC 1929-37. McGee's estimate for the total mass and diameter for the HI concentration directly centred upon NGC 1929-37 is: Mass, $5 \times 10^{6} M_{\odot}$; diameter, $350 \mathrm{pc}$. The maximum value of the brightness temperature is equal to $T_{\max }=100^{\circ} \mathrm{K}$, and the background temperature is of the order of $16^{\circ} \mathrm{K}$. The profile halfwidthafter subtraction of the background-is $22 \cdot 4 \mathrm{~km} / \mathrm{sec}$.

\section{Evolutionary Implications}

The association NGC 1929-37 is very probably a young star grouping embedded in a massive gaseous cloud. Westerlund (1961), on the basis of Limber's work (1960), assigns ages of the order of $2 \times 10^{6}$ years to groupings of this sort and it seems out of the question that the stars under consideration $\left(M_{V}<-4 \cdot 0\right)$ can be as old as $10^{7}$ years. The diameter of the star group is $150 \mathrm{pc}$ and the total estimated mass for the stars in the association is $2 \times 10^{4} M \odot$.

The stellar association is embedded in a hydrogen cloud with approximately twice the diameter of the stellar association. The estimated mass of the ionized hydrogen is between two and four times that of the stars, but the best value for the mass of the neutral atomic hydrogen is 100 times that of the ionized hydrogen!

The most important piece of further information that is needed to complete the picture is data on the radial velocity dispersion shown by the stars in the association. The stars in question are probably too close together and too much embedded in nebulosity to permit the measurement of their radial velocities by the objective prism technique, but a number of the brighter stars are accessible to the nebular spectrograph at Mount Stromlo Observatory. Optical data on polarization are obviously also very much wanted.

We should bear in mind that the total mass of the most rapidly evolving stars, those with $M_{V}<-4 \cdot 0$, amounts to only $4 \times 10^{3} M_{\odot}$, which means that there is a supply of ionized hydrogen sufficient for the formation of 10 to 15 generations of these stars; 
100 times as much as if we take into account the vast supply of neutral atomic hydrogen. It seems most likely that the proximity of young stars and plentiful hydrogen gas is not an accidental one - and we may look upon NGC 1929-37 as one of the places in our universe where star birth and evolution are continuing processes. On the basis of our present information, we may conclude that there is sufficient available hydrogen to permit continued star birth at the present rate for an interval of $10^{9}$ years or even $10^{10}$ years.

It is of course possible - though unlikely - that the stellar association is by chance seen passing through a vast gaseous cloud, or that it is by chance seen projected against such a cloud. The fact that NGC 1929-37 stands out clearly in Mathewson's contour diagram in paper 54 and in McGee's in paper 62 speaks against the assumption of a merely accidental and temporary association of gas and stars. We shall not consider this possibility in the present paper.

If we assume that the association of massive stars with ionized and neutral atomic hydrogen is not an accidental one, then there are three major alternatives for the dynamical and evolutionary status of Constellation I. I shall discuss first the hypothesis that I favour:

(a) The Stars in NGC 1929-37 are Part of an Expanding Association.-The Viria] Theorem permits us to calculate the expected velocity dispersion of individual stars and individual gas clouds that may be expected in a system with a total mass of $5 \times 10^{6} M_{\odot}$, with a radius of 175 pc $(21-\mathrm{cm}$ results $)$. We have

$$
\overline{V^{2}}=\frac{3 G M}{5 R}
$$

where (c.g.s. units):

$$
G=6 \cdot 7 \times 10^{-8}, M=10^{40}, R=6 \times 10^{20}
$$

and hence we find

$$
\sqrt{V^{2}}=8 \mathrm{~km} / \mathrm{sec} .
$$

This represents an upper limit, since not all of the gas may constitute part of a single dynamical unit. There could be little doubt that the stellar association is an expanding one if we should find an observed dispersion in random velocities for the stars of the order of $8 \mathrm{~km} / \mathrm{sec}$ or greater. The observed halfwidth of the $21-\mathrm{cm}$ feature observed by McGee $(22.4 \mathrm{~km} / \mathrm{sec})$ suggests a dispersion in gaseous random velocities somewhat, but not greatly, in excess of the critical figure of $\pm 8 \mathrm{~km} / \mathrm{sec}$, but one should bear in mind that the system as a whole may be stable, especially if the distribution of the gas is clumpy. I shall be in favour of considering the association as an expanding one if the observed dispersion in random velocities of the stars proves to be in excess of $\pm 5 \mathrm{~km} / \mathrm{sec}$.

If the average outward velocity of an escaping star is of the order of $5 \mathrm{~km} / \mathrm{sec}$, then escape will take place in times of the order of $2 \times 10^{7}$ years. The absence of an observed marked concentration of evolved stars seems to speak in favour of the hypothesis of continued star formation with escape from the association of the stars thus formed in intervals of less than $10^{8}$ years.

Since I shall ignore the hypothesis of a chance association of gas and stars, two alternative hypotheses deserve brief consideration. 
(b) The Stellar Association is a Stable One.-This hypothesis will deserve serious consideration if the observed dispersion in stellar random velocities proves to be less than $5 \mathrm{~km} / \mathrm{sec}$ - certainly if it is of the order of $2 \mathrm{~km} / \mathrm{sec}$ or less. In the course of time, the blue-white supergiants of the vertical main sequence will then obviously have to move away in the colour-magnitude array from their present conspicuous place with $M<-4.0$ and with $(B-V)_{0}=-0.3$ or less. They should presumably pass through a red supergiant stage before settling down as less conspicuous stars. These evolved blue-white supergiants will, on the second hypothesis, remain members of the association. There is strong observational evidence against the presence of large numbers of these evolved stars within the boundaries of the association and hence I reject this hypothesis.

Let us examine briefly the numbers of evolved stars that may be expected on this hypothesis. We observe about 60 stars with $M<-4 \cdot 0$, which belong to the inner part of the association and we may give $10^{7}$ years as a safe upper limit to the ages of these stars, with a smaller value, $2 \times 10^{6}$ years, representing their most probable average age. If star birth is a continuing process, and if the evolved stars are retained by the stable stellar aggregate, then a minimum number of 6000 evolved stars should accumulate in $10^{9}$ years, 10 times as many in $10^{10}$ years.

(c) The Massive Supergiant Stars now Observed Blow Up within $10^{7}$ Years of Their Birth.-This represents the most drastic of the evolutionary processes envisaged. Following each explosion, most of the gas from the star would presumably be returned to the gaseous clouds and the whole process would be a cyclical one. However, with each explosion the interstellar gas would presumably be enriched with heavier elements. The observational evidence presented by Faulkner (this volume, paper 64) does not suggest unusually great abundance of the heavier elements versus hydrogen and hence we reject this third hypothesis.

\section{Conclusions}

Our discussion shows how important it is that we should study further these young associations and their gaseous and stellar content. For the future, observations of stellar and gaseous radial velocities are clearly of great importance. More data on relative abundances for the gaseous clouds are also needed. Finally, it is obviously necessary to derive the colour-magnitude array for the stars to the faintest limits within reach and to check as best as can be done on the accuracy and reliability of the radio data for the HI and HII clouds that are associated with objects like NGC 1929-37.

Nowhere does there appear to be a better chance to see star birth and evolution at work than in the young associations of the Large Magellanic Cloud.

\section{References}

BlaAuw, A. (1958)._-“Stellar Populations." [Vatican City 1957.] (Ed. D. J. K. O'Connell.) (North Holland Publishing Co.: Amsterdam.)

Bok, B. J., and Bok P. F. (1962).-M.N. 124: 435-44= Mount Stromlo Obs. Reprint No. 67. Bor, B. J., Bor, P. F., and BAsinskx, J. M. (1962).-M.N. 123: 487-96 = Mount Stromlo Obs. Reprint No. 51.

Doherty, L., Henize, K. G., and Aller, L. H. (1956)-Ap. J. Suppl. 2: 345-63. 


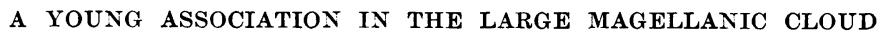

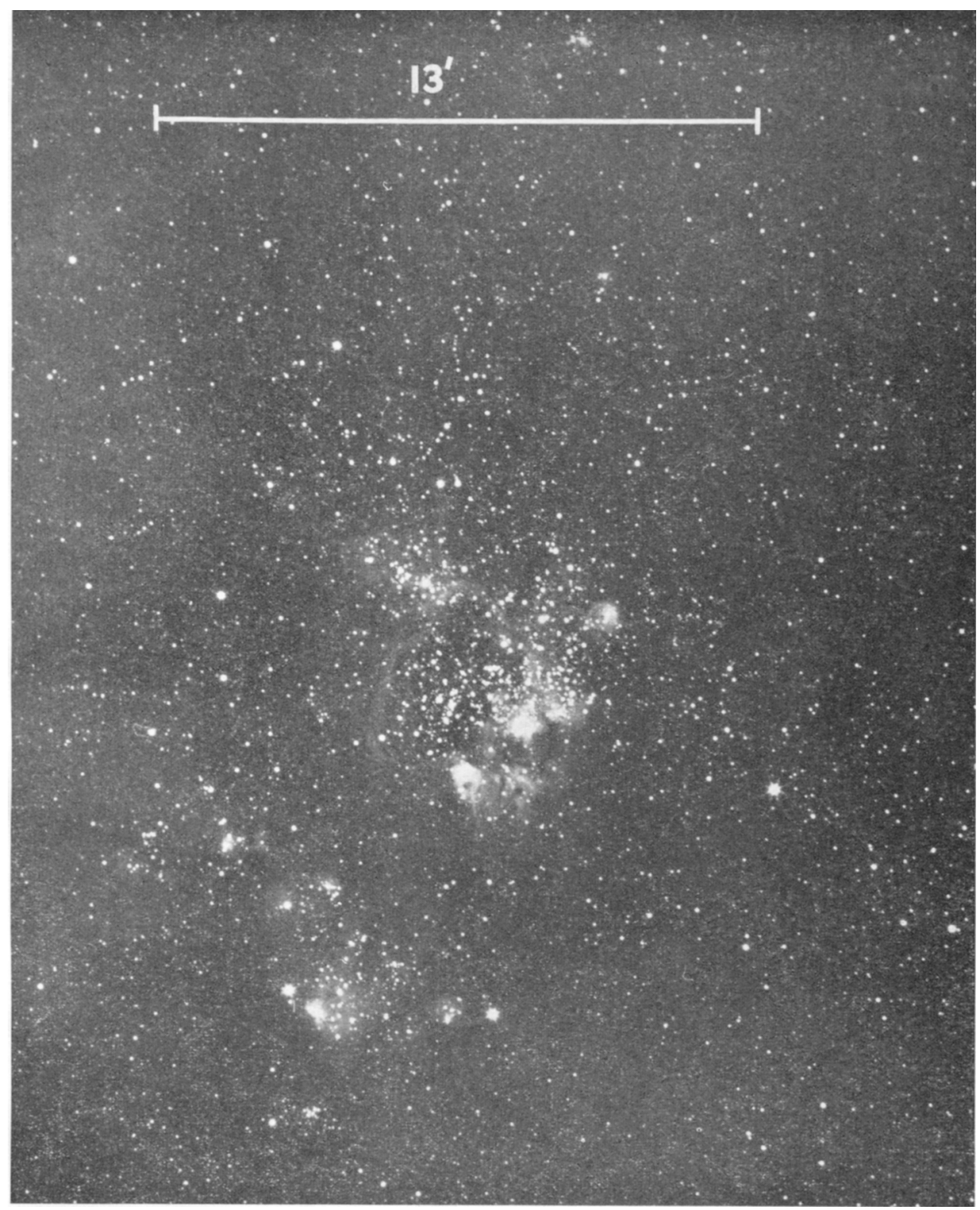

NGC 1929-34-35-36-37 photographed with the 74-inch reflector, 45-inch diaphragm, on a 103aO plate with a GG 13 filter, exposure time $90 \mathrm{~min}$. The white line at the top indicates 13', the approximate angular wirlth of the analysing beam at 21 -c'm wavelength for the Parkes 210 -foot radio telescope.

I.AC-CRSI Symposinm .No. 20, Paper tis 
Feast, M. W., Thackeray, A. D., and Wesselink, .A. J. (1960).-M.N. 121 : 337-85= Radcliffe

Obs. Comm. No. 55.

LIMBER, D. N. (1960).-Ap. J. 131 : 168-201.

Westerlund, B. E. (1961).-Uppsala Astron. Obs. Annaler 5 No. 1.

Note Added in Proof.-At the time of presentation of my paper at the Symposium, I had overlooked a paper by V. McK. Nail and H. Shapley (Proc. Nat. Acad. Sci. (Washington) 39: 358-62 (1953) (Harvard Obs. Reprint No. 373), which, to the best of my knowledge, is the first one to have stressed the prevalence of blue supergiant stars in the five named Constellations. The grouping NGC 1929-37 is called Constellation I and it is described as "a double aggregation".

\section{Discussion}

Eggen: If the expanding hypothesis is correct there should be a density gradient around the association.

Bok: There might very well be.

Davies: Does the large velocity dispersion $( \pm 10 \mathrm{~km} / \mathrm{sec})$ found by McGee for the neutral hydrogen mean that the gas clouds and associated stars form an unstable complex?

Bok: The halfwidth of the H-profile is $22 \cdot 4 \mathrm{~km} / \mathrm{sec}$, which means a dispersion in velocities close to the critical one for instability.

McGee: The halfwidth of the H-line profile $(22.4 \mathrm{~km} / \mathrm{sec})$ would indicate the presence of a single complex of neutral hydrogen in this direction.

de Vaucouleurs: When we discuss HI line widths we should remember that they represent an integration over the beamwidth and along the line of sight through the whole thickness of the Cloud, hence they are larger than in any given association.

Lindblad: If you looked for the stars escaped from the association you would not expect a spherical distribution. We have a strong rotational motion on which the peculiar motions are superimposed. The stars should therefore be spaced along a wide arc corresponding to what I have called a dispersion orbit.

Westerlund: The gas in the region is definitely expanding as judged from the shape of the emission features on 74-inch plates taken with an interference filter centred on $\lambda 5007$.

Tifft: Could you comment on the reddening in the region? With all the HI about one would expect quite a bit of dust and therefore reddening, or a very low dust to gas ratio.

Bok: The visual absorption is 0.7 but differs from one point to another.

Gascoigne: From the colours of some of the B stars in your region (around -0.25 or even bluer) I would have thought the reddening is less than the $A_{V}=0 \mathrm{~m} \cdot 7$ you quote.

Bok: It is an irregular field and some stars are unreddened but the average is close to $0 \mathrm{~m} 7$.

Westerhout: I am confused. If the gas is just as stirred up as the stars (dispersion about 8 or $10 \mathrm{~km} / \mathrm{sec}$ ) then it would walk out just as fast as the stars. Why then should we expect a large number of evolved stars around the region? The whole thing expands together. Therefore, if we do not find evolved stars in the association it means that star formation has just started. The only other possibility is that the gas is held together by another force. But after what we have heard about the magnetic field in our own Galaxy, which now seems too small to have much effect on the gas, one is hesitant to propose magnetic forces in the Clouds.

McGee: Allowing for bandwidth of the H-line receiver the HI velocity dispersion would be of the right order $(8$ or $9 \mathrm{~km} / \mathrm{sec})$.

Gascoigne: Dr. Arp, you have spoken of the importance of finding regions of actual star formation in your introductory address. Will you comment on this?

Arp: In the loose SMC cluster NGC 371, I have found (unpublished) that stars in different parts of the cluster, while all young, seem to differ slightly in age. I would interpret 
this to mean that star formation took place over an extended region which is nevertheless all associated into what looks like one big loose cluster. This is a much different case than in NGC 330 where star formation took place all within a very small region and therefore probably all at very much the same time.

Westerlund: There are several dense young clusters in the LMC where star formation appears to have continued in the centre after it was over in the outer parts.

\title{
69. THE WING OF THE SMALL MAGELLANIC CLOUD
}

\author{
B. E. Westerlund \\ Mount Stromlo Observatory
}

\section{The Clusters}

It is important to study some of the less bright and less rich clusters in the Magellanic Clouds in order to complete the picture of their populations as derived from studies of their giant clusters and associations. The region around NGC 419

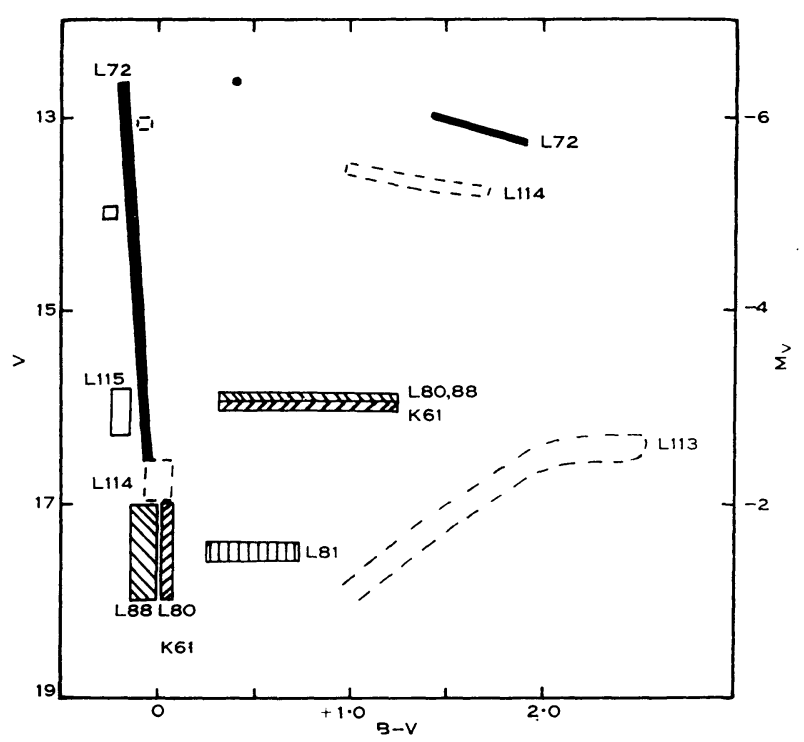

Fig. 1.-Composite colour-magnitude diagram for the blue clusters and for the globular cluster L113.

in the Small Cloud is fairly rich in small- and medium-sized blue clusters and has, in addition, a photoelectric sequence (Arp 1958). Several clusters and groups of stars in this region have now been investigated (Westerlund, unpublished data). The resulting colour-magnitude arrays for four clusters in this region are presented in Figure 1 together with the arrays for three clusters in the wing. The clusters are identified by their numbers in Kron's $(\mathrm{K})$ or Lindsay's (L) catalogues (Kron 1956; Lindsay 1958). 